\title{
Atuação em ensino, pesquisa e extensão no Herbário do Instituto de Biologia da Univer- sidade Federal do Rio de Janeiro
}

\author{
Performance in teaching, research and extension in the Herbarium of the Biology Institute of \\ the Federal University of Rio de Janeiro
}

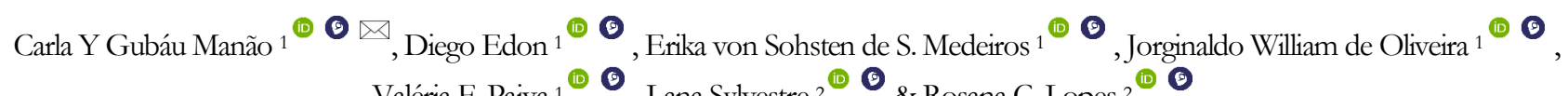
Valéria F. Paiva $1^{(1)}$, Lana Sylvestre $2^{(10)}$ \& Rosana C. Lopes $2^{(1)} \mathbf{0}$

1. Universidade Federal do Rio de Janeiro, Instituto de Biologia, Herbário RFA, Rio de Janeiro, Rio de Janeiro, Brasil

2. Universidade Federal do Rio de Janeiro, Instituto de Biologia, Curadoria do Herbário RFA, Rio de Janeiro, Rio de Janeiro, Brasil

Palavras-chave:

Biodiversidade. Coleções biológicas. Ducommun. Flora brasileira. Fungos. Occhioni. RFA.

Keywords

Biodiversity. Biological collections. Ducommun. Brazilian flora. Fungi. Occbioni. RFA.

Recebido em: 31/01/2021

Aceito em: 06/05/2021

Editor responsável: Cristiana B. N. Costa (UFSB)

eISSN: 2595-6752

\section{(c) (i)}

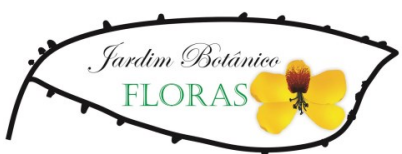

\section{Resumo}

O Herbário do Departamento de Botânica (RFA), do Instituto de Biologia pertencente à Universidade Federal do Rio de Janeiro, foi criado em 1954, pelo professor Paulo Occhioni, para atender às atividades de ensino e pesquisa. A coleção teve início a partir das coletas resgatadas na Faculdade de Medicina do Rio de Janeiro, representadas por 794 exsicatas da Coleção da Flora Europeia do botânico J.C. Ducommun. Posteriormente, a coleção foi incrementada com amostras da Flora do Rio de Janeiro, resultado de excursões botânicas periódicas. Atualmente, constam no herbário 45.260 espécimes de todos os grupos vegetais e fungos. Destacam-se 118 tipos nomenclaturais e duas coleções auxiliares, como a Carpoteca e a Fototeca. O RFA é uma coleção dinâmica que se preocupa com a divulgação científica e com as atividades que envolvem o ensino, a extensão e a pesquisa, principalmente por ser parte da universidade.

\begin{abstract}
The Herbarium of the Department of Botany (RFA) of the Institute of Biology from the Federal University of Rio de Janeiro was created in 1954 by Professor Paulo Occhioni in oder to support teaching and research activities. The herbarium had its origin from collections gathered at the Medical School of Rio de Janeiro and it was represented by 794 exsiccates from the European Flora Collection by the botanist J.C. Ducommun. Subsequently, the collection has been expanded with samples from Flora of Rio de Janeiro, acquired during periodic botanical expeditions. Currently, there are 45,260 specimens of plants and fungi in the herbarium. There are 118 nomenclatural types and two auxiliary collections, such as Carpotheque and Photo Library. RFA is a dynamic collection that focuses on scientific dissemination and other activities, such as teaching, extension and research, mainly because it is part of the University.
\end{abstract}

Introdução

As coleções biológicas são representadas por um conjunto de organismos, ou partes destes, preservados geralmente fora do ambiente natural, ex situ. As amostras são preparadas de acordo com protocolos específicos a cada coleção, organizados de modo a informar a procedência e a identificação taxonômica de cada espécime, o que lhe confere o status científico (Aranda, 2014). 
Entre as várias coleções biológicas, destacamos neste trabalho o Herbário, que é o termo designado para retratar uma coleção de plantas, de algas, de líquenes ou de fungos, desidratados. O Herbário é um importante repositório de biodiversidade, reconhecido por alguns autores como uma "máquina do tempo" ou uma "mina de ouro" de informações, com função principal de servir como um recurso essencial para estudos em taxonomia, sistemática, ecologia, anatomia, morfologia, conservação, biodiversidade, etnobotânica e paleobiologia (Funk, 2007; Marino, 2018; Gasper et al., 2020).

Atualmente, as informações das exsicatas contidas nos herbários vêm sendo aproveitadas de maneiras inovadoras, não previstas pelos colecionadores originais, mas aplicadas a um novo cenário, onde as coleções passam a ser essenciais para pesquisas avançadas que podem envolver temas como o controle da disseminação de doenças infecciosas ou até mesmo respostas para as mudanças climáticas e outros grandes desafios (Peixoto; Maia, 2013; Heberling; Isaac, 2017; Ríos-Saldaña et al., 2018). De acordo com os dados disponíveis no Catálogo da Rede Brasileira de Herbários $(\mathrm{RBH})$, existem no Brasil 200 herbários ativos, sendo que as quatro maiores coleções estão concentradas na região Sudeste (Herbário do Jardim Botânico do Rio de Janeiro (RB), ca. 800 mil amostras; Herbário do Museu Nacional (R), ca. 550 mil; Herbário Maria Eneyda P. K. Fidalgo (SP), ca. 497 mil) e Sul (Herbário do Museu Botânico Municipal (MBM), ca. 425 mil) (SBB, 2021).

O acervo do Herbário do Departamento de Botânica, Instituto de Biologia, da Universidade Federal do Rio de Janeiro (RFA), iniciou-se em 1954, no antigo curso de História Natural da Faculdade Nacional de Farmácia do Rio de Janeiro, sob a curadoria do professor Paulo Occhioni, que começou a organizar a coleção assim que assumiu a Cátedra de Botânica Aplicada à Farmácia.

A partir da recuperação dos materiais dos catálogos depositados em latas no antigo Herbário abandonado da Faculdade Nacional de Medicina, foi possível resgatar algumas coletas de Freire Alemão e a coleção da Flora Europeia do botânico Suíço J.C. Ducommun (1829-1892), a qual estava bem conservada. Acredita-se que esta doação teria sido adquirida por D. Pedro II (Occhioni, 1960-1962ab; Lopes; Oliveira, 2015). A coleção originalmente foi criada com o objetivo de servir como suporte didático às disciplinas de Botânica e acrescida, posteriormente, com materiais de excursões botânicas realizadas em diversas regiões fitogeográficas do Brasil e intercâmbio entre herbários. Devido a inúmeras reformas no ensino, a guarda dessa coleção foi transferida várias vezes e, atualmente, encontra-se no Instituto de Biologia.

Desde 1978, o Herbário RFA é reconhecido no Index Herbariorum, catálogo mundial de herbários, coordenado pelo Jardim Botânico de Nova York (Thiers, 2016).

O RFA participou de importantes editais, com os projetos "Herbário RFA: Organização, Ampliação, Informatização, Manutenção e Criação de Herbário Virtual Infraestrutura” (2004-2007) e "Herbário RFA: Infraestrutura" (2005-2007), que tiveram o apoio financeiro do Conselho Nacional de Desenvolvimento Científico e Tecnológico (CNPq) e da Fundação de Amparo à Pesquisa do Estado do Rio de Janeiro. Com o auxílio das agências de fomento foi possível fazer melhorias nas condições de preservação da coleção.
O RFA participou da Rede Fluminense de Herbários do Rio de Janeiro. Neste período, com a aprovação do "Projeto de Informatização dos Herbários Fluminenses”, submetido à FAPERJ, foi possível adquirir equipamentos de informática, lupa e armários para acondicionar a coleção. Hoje, o RFA integra a Rede Brasileira de Herbários, onde parte de seus dados estão disponíveis na página da Sociedade Botânica do Brasil (SBB, 2021).

Em 2016, o Herbário RFA passou a integrar o programa RE FLORA (http://reflora.jbrj.gov.br), onde, por um ano, contou com um bolsista para o trabalho de informatização dos dados e, em 2018, aderiu ao projeto "Instituto Nacional de Ciência e Tecnologia (INCT) - Herbário Virtual da Flora e dos Fungos do Brasil" (http:/ / splink.cria.org.br/), com o objetivo de aumentar a visibilidade e o acesso público da coleção.

Neste estudo, o RFA visa mostrar a importância do seu acervo e apresentar as suas principais atividades e contribuições no ensino, na pesquisa, na extensão e para a Flora Brasileira.

\section{Material e Métodos}

Foi feito o levantamento das atividades desenvolvidas no Herbário RFA, que inclui o suporte ao ensino e à pesquisa desde a sua origem. Recentemente, nos últimos cinco anos e com a formação de uma nova equipe, as ações de extensão foram impulsionadas e passaram a fazer parte da rotina do herbário.

Na pesquisa, foi avaliada a atuação do herbário RFA como um importante instrumento para o desenvolvimento da ciência e, em parte, para o reconhecimento da biodiversidade da Flora Brasileira. A informatização dos dados da coleção teve início a partir de 2009 e, posteriormente, a sua divulgação nas plataformas do JABOT e na rede Species Link, que possibilitaram o uso de ferramentas que facilitaram a obtenção dos resultados tratados neste estudo.

Independente dos tipos de projetos, toda amostra botânica incorporada na coleção segue as normas do "Regimento do Herbário RFA do Departamento de Botânica, Instituto de Biologia da Universidade Federal do Rio de Janeiro" e particularidades referentes à preservação da amostra, conforme indicado no manual de técnicas de Fidalgo e Bononi (1989).

\section{Resultados e Discussão}

\section{A Coleção Botânica do RFA}

Constam atualmente no herbário RFA 45.260 espécimes de todos os grupos vegetais e fungos. De 2015 a 2020, o número de tipos nomenclaturais na coleção quase quadruplicou, representados por 64 amostras de fungos, 52 angiospermas, uma samambaia e uma alga (Lopes; Oliveira, 2015) (Tabela 1). Embora o RFA possua amostras de países europeus e latino-americanos originárias de permutas, que representam $7 \%$ da coleção, a maior representatividade está nas amostras da Flora do Estado do Rio de Janeiro, que perfazem $46 \%$, geralmente coletadas fora de Unidades de Conservação, totalizando 21.016 exsicatas. 
Tabela 1. Lista dos Typus nomenclaturais do Herbário RFA, do Departamento de Botânica, do Instituto de Biologia/UFRJ. (O número dois se refere à quantidade de duplicatas).

\begin{tabular}{|c|c|c|}
\hline Grupo / Família / Espécie & Classificação & $\begin{array}{c}\text { Coletor e } \\
\mathrm{N}^{\circ} \text { de Coleta }\end{array}$ \\
\hline \multicolumn{3}{|l|}{ ALGA } \\
\hline \multicolumn{3}{|l|}{ CHORDARIACEAE } \\
\hline \multicolumn{3}{|l|}{ ANGIOSPERMAS } \\
\hline \multicolumn{3}{|l|}{ APOCYNACEAE } \\
\hline Oxypetalum kublmannianum Occhioni & Isótipo & Amaro Macedo s.n. (RFA 7.315) \\
\hline Oxypetalum marambaiense Occhioni & Holótipo & P. Occhioni 4.184 \\
\hline Oxypetalum pauciflorum Occhioni & Holótipo & Nagib Saddi 403 \\
\hline \multicolumn{3}{|l|}{ ASPARAGACEAE } \\
\hline Clara gracilis R.C.Lopes \& Andreata & Parátipo & R.C. Lopes 151 \\
\hline \multicolumn{3}{|l|}{ BROMELIACEAE } \\
\hline Canistrum montanum Leme & Parátipo & A.P.G. Faria 201 \\
\hline Orthophytum itambense Versieux \& Leme & Isótipo & L.M. Versieux 149 \\
\hline \multicolumn{3}{|l|}{ CALOPHYLLACEAE } \\
\hline Kielmeyera aureovinosa M. Gomes & Holótipo & M. Gomes 1.053 \\
\hline Kielmeyera rubriflora var. affinis Saddi & Isótipo & Gardner 3.612 \\
\hline \multicolumn{3}{|l|}{ CAMPANULACEAE } \\
\hline Lobelia brasiliensis A.O.S.Vieira \& G.J.Sheph. & Parátipo & A. Mattos Filho 350 \\
\hline \multicolumn{3}{|l|}{ CUCURBITACEAE } \\
\hline Cayaponia rugosa Gomes-Klein \& Pirani & Isótipo & A.P. Duarte 10.643 \\
\hline \multicolumn{3}{|l|}{ ERIOCAULACEAE } \\
\hline Tonina fluviatilis Aubl. & Parátipo & D.A. Folli 1.855 \\
\hline \multicolumn{3}{|l|}{ FABACEAE } \\
\hline Cassia spinigera Rizzini & Isótipo & A. P. Duarte 13.973 \\
\hline Machaerium hoehneanum Ducke & Parátipo & A. Ducke s.n. (RFA 17.755) \\
\hline Mimosa manidea Barneby & Parátipo & A.P. Duarte 10.677 \\
\hline Senegalia duartei Seigler \& Ebinger & Isótipo & A. P. Duarte 5.764 \\
\hline Stryphnodendron confertum Heringer \& Rizzini & Isótipo & E.P. Heringer 9.178 \\
\hline Stryphnodendron consimile Mart. & Holótipo & F.B. Ramalho 52 \\
\hline Stryphnodendron duckeanum Occhioni & Isótipo & A. Ducke s.n. (RFA 11.684) \\
\hline Stryphnodendron bumile Martins & Holótipo & E.P. Heringer 7.783 \\
\hline Stryphnodendron piptadenioides E.M.O.Martin & Holótipo & D.P. Lima 13.302 \\
\hline \multicolumn{3}{|l|}{ LAMIACEAE } \\
\hline Hesperozygis dimidiata Epling \& Mathias & Isótipo & R. Reitz 5.450 \\
\hline \multicolumn{3}{|l|}{ MALPIGHIACEAE } \\
\hline Heteropterys fragilis Amorim & Parátipo & P. Occhioni 6.631 \\
\hline Heteropterys occhionii Amorim & Holótipo & P. Occhioni 8.213 \\
\hline Peixotoa cipoana C.E.Anderson & Holótipo & P. Occhioni 5.009 \\
\hline Peixotoa cipoana C.E.Anderson & Parátipo & P. Occhioni 5.054 \\
\hline \multicolumn{3}{|l|}{ MALVACEAE } \\
\hline Byttneria schumannii Cristóbal & Isótipo & C. Pereira 142 \\
\hline MORACEAE & & \\
\hline Brosimum mello-barretoi Standl. & Isótipo & M. Barreto 1.658 \\
\hline Dorstenia bonijesu Carauta \& C.Valente & Isótipo & C.M. Rizzini 217 \\
\hline Dorstenia bonijesu Carauta \& C.Valente & Parátipo & V.F. Ferreira 1.729 \\
\hline Dorstenia drakena $\mathrm{L}$ & Lectótipo & Velloso s.n. (RFA 3.961) \\
\hline MYRTACEAE & & \\
\hline Myrcia dichrophylla D.Legrand & Parátipo & R. Reitz 3.061 \\
\hline Myrciaria hatschbachii Mattos & Isótipo & G. Hatschbach 7.662 \\
\hline OLACACEAE & & \\
\hline Liriosma adhaerens Spruce ex Engl. & Isótipo & E. Pereira 284 \\
\hline OPILIACEAE & & \\
\hline Agonandra fluminensis Rizzini \& Occhioni & Holótipo & P. Occhioni 7.609 \\
\hline Agonandra fluminensis Rizzini \& Occhioni & Parátipo & Rizzini 7.712 \\
\hline Agonandra fluminensis Rizzini \& Occhioni & Parátipo & Rizzini 7.713 \\
\hline OXALIDACEAE & & \\
\hline Oxalis monochasiata Fiaschi & Isótipo & L.P. Queiroz 10.699 \\
\hline PICRAMNIACEAE & & \\
\hline Picramnia monochlamydea Occhioni \& Rizzini & Holótipo & P. Occhioni 6.027 \\
\hline Picramnia monochlamydea Occhioni \& Rizzini & Parátipo & P. Occhioni 6.050 \\
\hline POLYGALACEAE & & \\
\hline Polygala irwinii Wurdack & Parátipo & G. Hatschbach 32.027 \\
\hline RUBIACEAE & & \\
\hline Borreria dichasia Sucre \& C.G.Costa & Isótipo & E. Pereira 5.158 \\
\hline
\end{tabular}


Tabela 1. Lista dos Typus nomenclaturais do Herbário RFA, do Departamento de Botânica, do Instituto de Biologia/UFRJ. (O número dois se refere à quantidade de duplicatas).

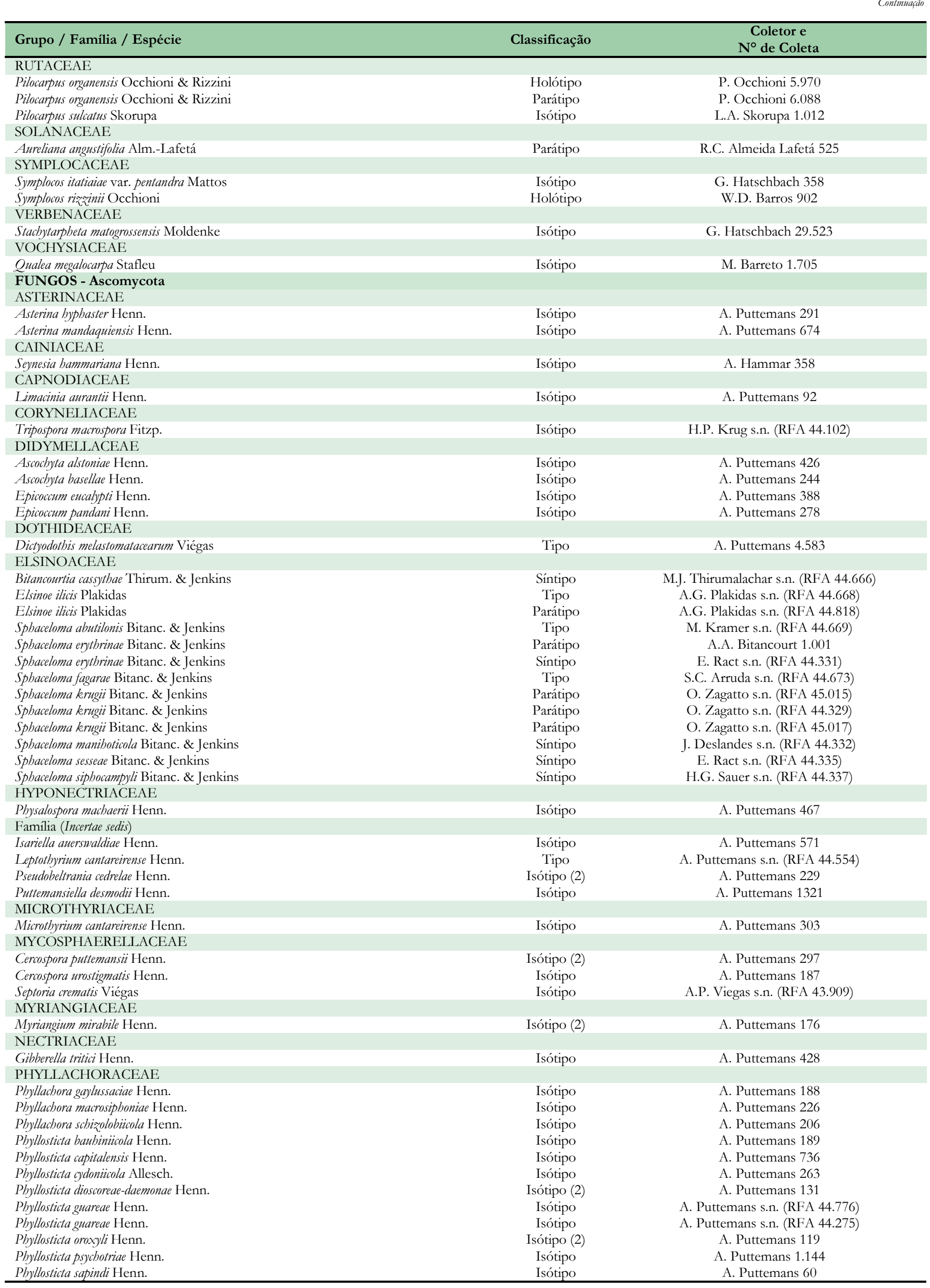


Tabela 1. Lista dos Typus nomenclaturais do Herbário RFA, do Departamento de Botânica, do Instituto de Biologia/UFRJ. (O número dois se refere à quantidade de duplicatas).

\begin{tabular}{|c|c|c|}
\hline Grupo / Família / Espécie & Classificação & $\begin{array}{c}\text { Coletor e } \\
\mathbf{N}^{\circ} \text { de Coleta }\end{array}$ \\
\hline \multicolumn{3}{|l|}{$\overline{\text { PLEOSPORACEAE }}$} \\
\hline \multirow{2}{*}{\multicolumn{3}{|c|}{ POLYSTOMELLACEAE }} \\
\hline & & \\
\hline Dotbidella mikaniae Henn. & Isótipo & A. Puttemans 230 \\
\hline \multicolumn{3}{|l|}{ TRIBLIDIACEAE } \\
\hline Pseudographis cocoes Henn. & Isótipo & A. Puttemans 205 \\
\hline Pseudographis cocoes Henn. & Isótipo & A. Puttemans s.n. (RFA 44.410) \\
\hline \multicolumn{3}{|l|}{ TUBEUFIACEAE } \\
\hline Puttemansia lanosa Henn. & Isótipo & A. Puttemans 178 \\
\hline \multicolumn{3}{|l|}{ XYLARIACEAE } \\
\hline Ustulina brasiliensis Speg. & Isótipo & Puiggari 1.504 \\
\hline \multicolumn{3}{|l|}{ FUNGOS - Basidiomycota } \\
\hline \multicolumn{3}{|l|}{ FOMITOPSIDACEAE } \\
\hline Phaeolus subbulbipes (Hfalenn.) O. Fidalgo \& M. Fidalgo & Isótipo & A. Puttemans s.n. (RFA 44.412) \\
\hline \multicolumn{3}{|l|}{ PUCCINIACEAE } \\
\hline Puccinia puttemansii Henn. & Isótipo (2) & A. Puttemans 140 \\
\hline \multicolumn{3}{|l|}{ Família Incertae sedis } \\
\hline Aecidium piptocarphae Henn. \& H.K.G. & Tipo & A. Puttemans s.n. (RFA 44.543) \\
\hline Aecidium puttemansianum Henn. & Isótipo & A. Puttemans 676 \\
\hline Uredo chrysophyllicola Henn. & Isótipo & A. Puttemans 153 \\
\hline Uredo puttemansii Henn. & Isótipo & A. Puttemans 197 \\
\hline \multicolumn{3}{|l|}{ SAMAMBAIA } \\
\hline \multicolumn{3}{|l|}{ MARSILEACEAE } \\
\hline Marsilea crotophora D.M.Johnson & Isótipo & G. Hatschbach 30.470 \\
\hline
\end{tabular}

A coleção está representada por 83,31\% de Angiospermas, 9,08\% de Algas, 4,90\% de Fungos, 2,21\% de Samambaias e Licófitas, $0,47 \%$ de Briófitas e menos de $0,03 \%$ de Gimnospermas.

As dez famílias com maior representatividade no RFA são Fabaceae (com 9,74\% amostras), Asteraceae (5,91\%), Rubiaceae $(4,52 \%)$, Myrtaceae (3,10\%), Melastomataceae (2,93\%), Lamiaceae $(2,80 \%)$, Dictyotaceae (2,31\%), Poaceae (2,16\%), Apocynaceae $(2,12 \%)$ e Solanaceae $(2,09 \%)$, que perfazem juntas $37,68 \%$ da coleção. A maioria das famílias citadas é de angiospermas, que também apresentam grande diversidade para o bioma Mata Atlântica. Apenas Dictyotaceae não se insere neste grande grupo, por ser uma alga parda.

Ilustres botânicos que contribuíram com publicações sobre a flora brasileira foram vitais para o incremento do herbário RFA (Figura 1). Mesmo sendo uma coleção pequena, esta possui sua singularidade, principalmente por apresentar uma importante coleção de tipos de fungos e devido aos projetos vinculados à coleção.

As primeiras coletas que deram início ao RFA foram as de Freire Alemão e de J.C. Ducommun, sendo representadas atualmente por duas e 794 exsicatas, respectivamente. Estes números se devem ao fato que, na ocasião do resgate das coleções por P. Occhioni na Faculdade Nacional de Medicina do Rio de Janeiro, a coleção de Freire Alemão estava totalmente degradada, o que não ocorreu com a Flora Europeia do botânico suíço J.C. Ducommun (Occhioni, 1960-1962ab).

Posteriormente, a coleção foi incrementada com amostras da Flora Brasileira, resultantes de excursões botânicas periódicas realizadas pelo próprio curador, P. Occhioni, com acréscimo de 7.218 amostras, e com coletores como F. Segadas-Vianna - importante ecólogo brasileiro que criou o Departamento de Ecologia no Museu Nacional e, em seguida, na UFRJ, contribuiu com 420 amostras
- e a taxonomista C.M. Rizzini com 308, durante o seu período como curadora da coleção (Figura 1). Não menos importante, as doações e permutas recebidas também compõem este importante acervo aberto a visitações. Quanto à coleção de fungos e seus tipos nomenclaturais, mais de 1.500 amostras foram adquiridas durante a atuação do professor Verlande Duarte Silveira (1909-1991), na instituição. Este importante engenheiro agrônomo também foi curador do Herbário Micológico Arsène Puttemans e devido a sua grande contribuição na área de ensino, foi homenageado em 1984 e 1991 pela Sociedade Brasileira de Fitopatologia (Faria et al. 2020; Lima et al., 2019).

O RFA conta com duas coleções auxiliares, a Carpoteca (33 amostras) e a Fototeca (128 imagens). Embora representadas por um número de amostras reduzidas, estes materiais já foram de grande importância, principalmente quando o acesso a coleções no exterior era mais difícil. A internet só começou a se popularizar a partir da década de 1990, quando os primeiros bancos de dados de herbários começaram a ser disponibilizados.

Pesquisa

Em prol do ensino e da pesquisa da UFRJ para o mundo, a coleção do Herbário RFA apoia projetos desenvolvidos por alunos da graduação, da pós-graduação e pelos pesquisadores do Instituto de Biologia (IB), do Instituto de Microbiologia Prof. Paulo de Góes (IMPPG), do Instituto de Pesquisas de Produtos Naturais (IPPN), do Instituto de Química (IQ), da Faculdade de Farmácia (FF), da Faculdade de Medicina (FM), da Pós-Graduação do Museu Nacional e do Núcleo em Ecologia e Desenvolvimento Sócio-Ambiental de Macaé (NUPEM).

A cooperação do RFA em projetos com outras instituições também foi um dos fatores que propiciaram o crescimento da coleção. A parceria com a Extracta Moléculas Naturais S/A (de 2000 até 
Figura 1. Os 10 coletores brasileiros e estrangeiros famosos por suas pesquisas na área da Botânica, somando um total de 12.933 exsicatas (próximo a $30 \%$ da coleção geral do RFA).

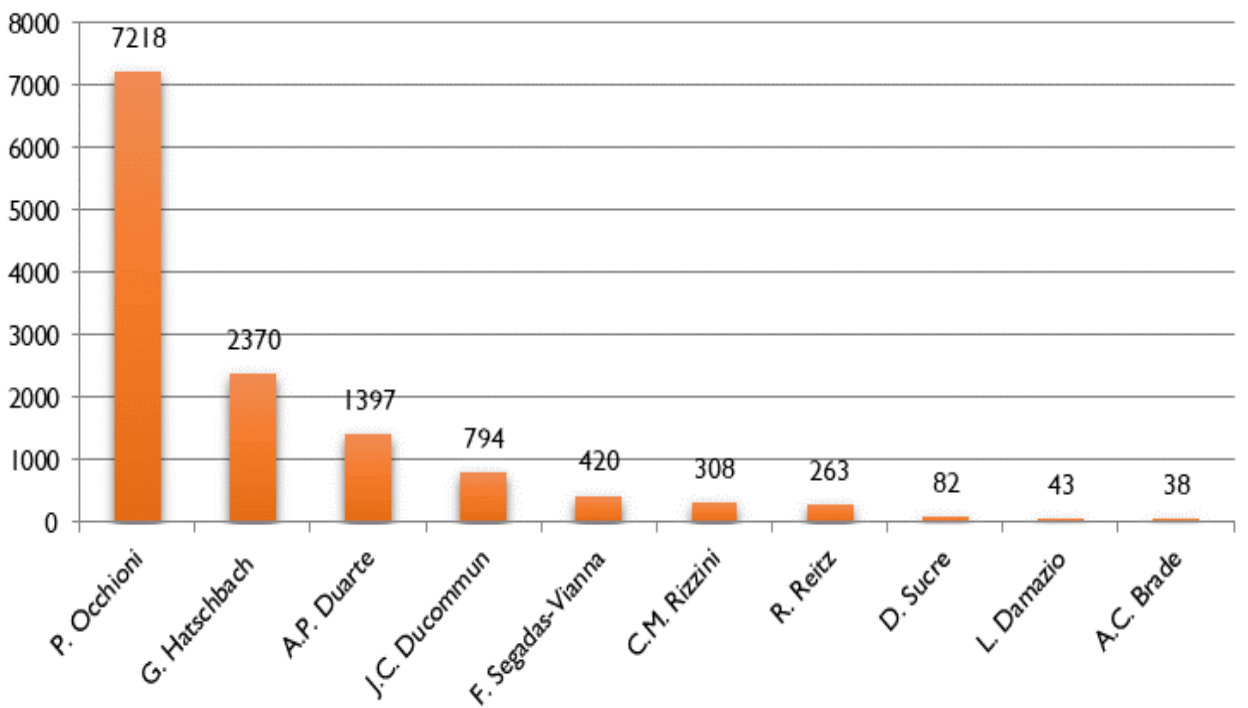

Figura 2. Atividades desenvolvidas pela equipe do Herbário RFA. a: Participação no evento LabAmigo, organizado pelos alunos de graduação durante a BioSemana UFRJ. b: Montagem de exsicatas com as alunas do projeto Jovens Talentos. c: Palestras realizadas durante o aniversário do herbário. d: Oficina Procedimentos de Herborização realizada no Jardim Didático.

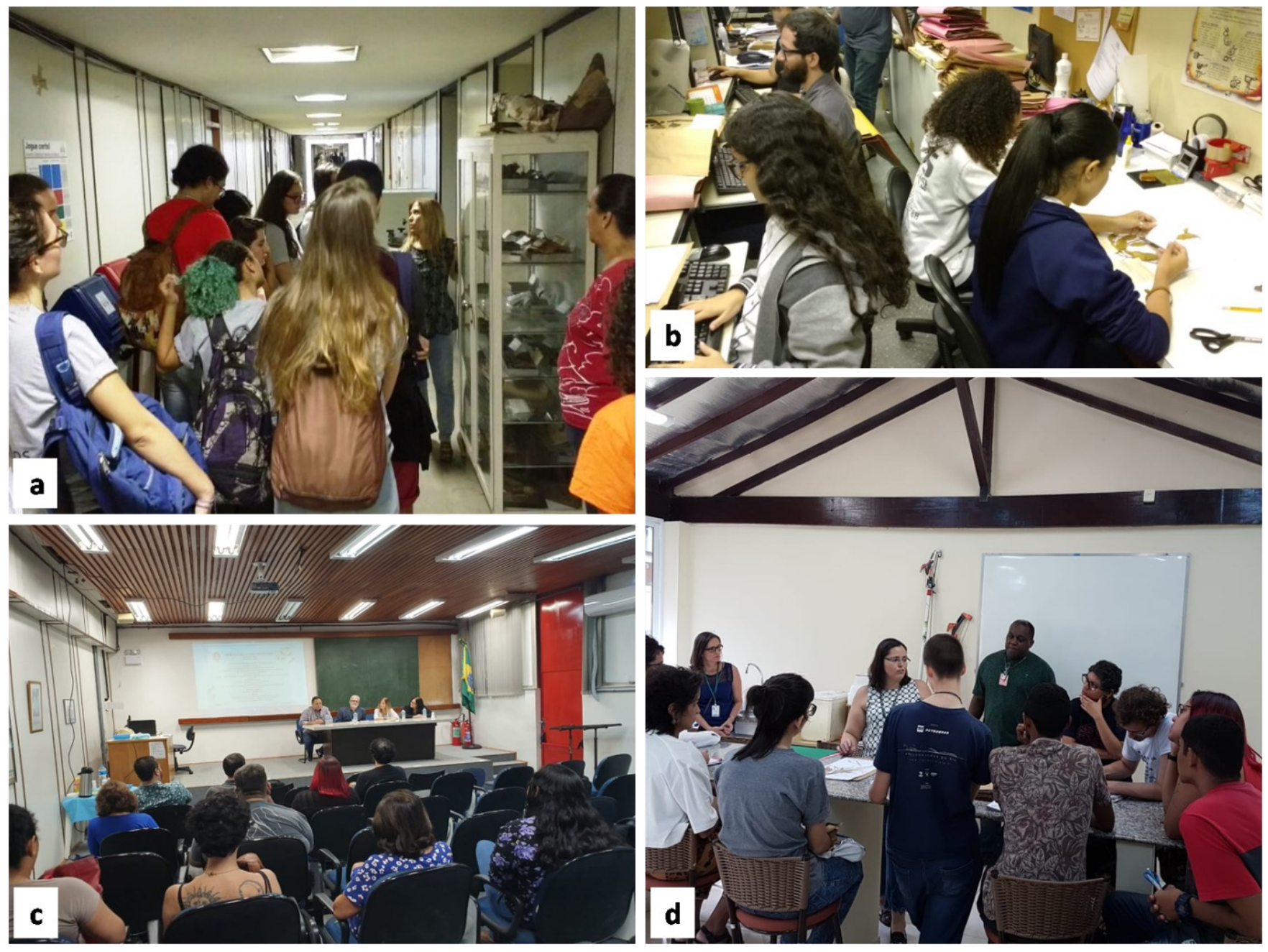


2004) possibilitou um acréscimo de 4.407 exsicatas de espécies nativas, também utilizadas para o preparo de importantes extratos nos estudos farmacêuticos. As amostras estão atualmente armazenadas no Laboratório de Extratos do Instituto de Pesquisas de Produtos Naturais (IPPN), localizado no Centro de Ciências da Saúde da UFRJ. Desde 2013, a colaboração com a Fiocruz gerou um acréscimo de aproximadamente 300 exsicatas originárias dos projetos: "Pesquisa de espécies vegetais superiores com atividades imunorreguladora e antiviral", "Avaliação do perfil químico e farmacológico de extratos e frações das partes aéreas de Malpighia glabra L.", "Plantas da Itaipu Binacional Refúgio Biológico Bela Vista Ervanário Plantas Medicinais, Foz do Iguaçu”, "APL Petrópolis", "Plantas Medicinais do Fórum Itaboraí/Fiocruz-Petrópolis" e "Aroeira Novos Tempos, Novos Rumos", todos associados ao uso de espécies nativas e saberes tradicionais.

Com essas contribuições durante toda existência desta coleção, é possível observar que os herbários, em geral, são essenciais por permitirem a documentação permanente de importantes exsicatas, que podem estar associadas a importantes patentes biotecnológicas ou a diferentes flórulas que se modificam ao longo do tempo, tanto pela ação antrópica ou por efeitos adversos, como perturbações naturais que alteram a cobertura vegetal (Fagundes; Fortes, 2006).

Ensino

Nas salas de aula, especificamente nas turmas de botânica dos cursos de Ciências Biológicas e de Farmácia, a equipe do herbário desenvolve junto com os professores da UFRJ e com os alunos todas as atividades que envolvem a coleta do material botânico e os demais procedimentos de herborização. Anualmente são atendidos cerca de 300 alunos, matriculados em oito disciplinas, compreendidas em 11 turmas, alem das solicitações ocasionais. A partir desta atividade, o aluno desperta para o conhecimento da botânica e começa a compreender a importância dos herbários no contexto da biodiversidade e demais áreas multidisciplinares. É importante ressaltar que um herbário é também um forte instrumento didático para o treinamento de estudantes e técnicos no reconhecimento da flora de um determinado local ou região (Fagundes; Fortes, 2006).

Extensão

Como partes da rotina do RFA são comuns atividades que envolvem o atendimento ao público externo, incluindo escolas e alunos de universidades. Contabiliza, desde 1989 até o ano de 2020, mais de 1.100 pessoas, brasileiras e estrangeiras, que visitaram o herbário, seus nomes e interesses específicos estão indicados nos livros de registro de visitantes.

Para atender a um público maior e mais variado, a equipe do RFA conta com projetos de extensão, que englobam o preparo de materiais educacionais para crianças e jovens, disponibilizados presencialmente em atividades externas ou por meio digital.

Dentre as atividades de extensão, ocorrem visitas guiadas de escolas, "Herbário de portas abertas" (período de integração dos calouros), "Aniversário RFA" (ciclo de palestras, oficinas ou minicursos), "LabAmigo" (visitação ao herbário durante a BioSemana UFRJ, uma parceria com o Centro Acadêmico de Ciências Biológicas), "Jovens Talentos" (atividades dos alunos de ensino médio no
RFA), "Oficina da coleta ao preparo de exsicatas" (para cursos de graduação), "Vivência no RFA" (complementação da carga horária de graduandos no RFA), "Uns minutinhos com o RFA" (vídeos informativos criados para esclarecer dúvidas dos alunos durante $\mathrm{O}$ estágio no RFA) e "Revistinha RFA" (um novo projeto que visa simplificar para as crianças e os jovens a importância das coleções botânicas, com desenvolvimento de atividades lúdicas) (Figura 2).

A equipe que integra o herbário RFA, mantém-se atualizada e sempre disposta a criar subsídios que possam auxiliar no ensino, pesquisa e extensão. A participação nas redes sociais como no Facebook (@Herbario.rfa) e no Instagram (herbariorfa.ufrj), auxilia na divulgação científica de forma popular, todo o trabalho aqui realizado.

\section{Conclusão}

Desmistificando o antigo senso comum de que as coleções estão associadas apenas a depósitos, conclui-se que o herbário é uma coleção dinâmica muito importante. Ele nos auxilia a entender sobre o passado por meio da consulta de coletas históricas, retrata o presente quando continuamos a incrementar o acervo e auxilia na resolução de problemas no futuro devido ao banco de dados produzido ao longo de muitas vidas. Além disso, tem um papel importante na divulgação da ciência e na formação de novos cientistas.

A divulgação científica é um importante recurso que auxilia no sucesso das atividades que envolvem o ensino, a pesquisa e a extensão, sendo uma das metas que a equipe se empenha em cumprir.

Considerando a relevância das coleções botânicas, podemos sugerir mais zelo, colaboração pública e privada para a manutenção dos acervos. Há aqueles que sugerem que uma exsicata poderia ser monetizada, no valor simbólico de, no mínimo, dez dólares. No entanto, não podemos esquecer que cada exemplar é singular. Como exemplos, não é possível valorar uma coleta histórica realizada no século XVIII por um importante naturalista, ou que seja originaria de uma região que hoje é desmatada, ou mesmo indicar o valor de uma exsicata representada por uma espécie hoje extinta. Por isso, esse patrimônio científico conta com acervo de valor inestimável.

\section{Agradecimentos}

Os autores agradecem a todos os alunos voluntários e bolsistas, do ensino médio e de graduação, que contribuíram durante os seus períodos de estágio, na manutenção e informatização do acervo, ao INCT - Herbário Virtual da Flora e dos Fungos, que disponibilizou uma bolsa de apoio técnico para o RFA, e ao Programa REFLORA do Instituto de Pesquisas Jardim Botânico do Rio de Janeiro.

\section{Financiamento}

À Fundação Carlos Chagas Filho de Amparo à Pesquisa no Estado do Rio de Janeiro (FAPERJ), pelo Edital 08/2020 - Programa Apoio à Conservação da Biodiversidade: Coleções Biológicas do Estado do Rio de Janeiro - 2020 (COLBIO), que irá custear o projeto "Modernização e Ampliação do Herbário RFA, preservando para gerar e divulgar conhecimento" ( ${ }^{\circ}$ de referência: 259077).

\section{Contribuições de Autoria}

Conceitualização: CYGM, RCL. Curadoria de dados: CYGM, DE, EVSDSM, JWDO, VFP, LS, RCL. Análise formal: CYGM, RCL. Aquisição de financiamento: CYGM, LS, RCL. Investigação: CYGM, EVSDSM, VFP, RCL. Metodologia: CYGM, DE, EVSDSM, JWDO, VFP, LS, RCL. Administração do projeto: CYGM, RCL. Recursos: CYGM, JWDO, LS, RCL. Programas: CYGM. Supervisão: CYGM. Validação: 
CYGM. Visualização: CYGM, DE, EVSDSM, JWDO, VFP, LS, RCL. Redação rascunho original: CYGM. Redação - revisão e edição: CYGM, DE, EVSDSM, JWDO, VFP, LS, RCL.

\section{Conflito de Interesse \\ Os autores declaram não haver conflitos de interesse a informar.}

\section{Disponibilidade dos Dados}

Os dados integrais analisados durante o estudo atual podem ser consultados na página http://rfa.jbri.gov.br/.

\section{Conformidade ética}

Não se aplica.

\section{Referências}

Aranda AT. Coleções biológicas: Conceitos básicos, curadoria e gestão, interface com a biodiversidade e saúde pública. In: Soares LMS, Lírio EJ, Pinheiro RFM, organizadores. III Simpósio sobre a Biodiversidade da Mata Atlântica [Internet]; 2014 maio 30 - jun 1; Rio de Janeiro, Brasil. Rio de Janeiro: Corbã Artes Gráficas; 2014 [citado em 17 maio 2021]. p. 45-56. Disponível em http:// www.sambio.org.br/simbioma/simbioma\%20iii/03.pdf

Fagundes JA, Fortes CE [Internet]. Herbário escolar: suas contribuições ao estudo da Botânica no Ensino Médio; 2006. [citado em 18 maio 2020]. Disponível em: http:// www.diaadiaeducacao.pr.gov.br/portals/pde/arquivos/16758.pdf

Faria CMX, Inácio, CA. Herbário Fitopatológico da UFRRJ "Verlande Duarte Silveira" - Elo da História da Fitopatologia no Brasil. Rodriguésia 2020;71:1-10. doi: 10.1590/21757860202071103

Fidalgo O, Bononi VLR. Técnicas de coleta, preservação e herborização de material botânico. São Paulo: Instituto de Botânica/ Imprensa Oficial do Estado de São Paulo; 1989.

Funk VA [Internet]. 100 Uses for a herbarium; 2007. [citado em 15 abr 2020]. Disponível em: http://www.virtualherbarium.org/ vh/100UsesASPT.html

Gasper AL, Stehmann JR, Roque N, Bigio NC, Sartori ÂLB, Grittz GS. Brazilian herbaria: an overview. Acta Botanica Brasilica 2020;34(2):352-359. doi: 10.1590/0102-33062019abb0390

Heberling JM, Isaac BL. Herbarium specimens as exaptations: New uses for old collections. American Journal of Botany 2017;104:963-965.

Lima MF, Carvalho RCP, Brioso, PST [Internet] Histórico da Sociedade Brasileira de Fitopatologia (SBF); 2019. [citado em 19 jun 2020]. Disponível em: http://sbfitopatologia.org.br/index.php? page $=$ sociedade

Lopes RC, Oliveira JW. Herbário da Universidade Federal do Rio de Janeiro, Rio De Janeiro (RFA). Unisanta Bioscience 2015;4 (6):399-401.

Marino, D [Internet]. The power of herbaria: a time machine for plant biology (2018). [citado em 15 abr 2020]. Disponível em: https://mappingignorance.org/2018/10/15/the-power-ofherbaria-a-time-machine-for-plant-biology-research/\#note-55881

Occhioni P. Herbário da Faculdade Nacional de Farmácia da Universidade do Brasil. Anais da Faculdade Nacional de Farmácia 1960-1962a;XII-XIII-XIV(7):93-95.

Occhioni P. Catalogo do Herbário da Faculdade Nacional de Farmácia. In: Anais da Faculdade Nacional de Farmácia 1960_1962b;XII-XIII-XIV: 7:97-98.

Peixoto AL, Maia LC (organizadoras). Manual de procedimentos para herbários. Recife: Editora Universitária UFPE; 2013.

Ríos-Saldaña CA, Delibes-Mateos M, Ferreira CC. Are fieldwork studies being relegated to second place in conservation science?
Global Ecology and Conservation 2018;14. doi: 10.1016/ j.gecco.2018.e00389

SBB (Sociedade Botânica do Brasil) [Internet] Catálogo da Rede Brasileira de Herbários (2021). [citado em 28 abr 2021]. Disponível em: https://www.botanica.org.br/a-rede-brasileira-deherbarios/

Thiers B. [Internet] Index Herbariorum: A Global Directory of Public Herbaria and Associated Staff. New York Botanical Garden's Virtual Herbarium 2016. [citado em 15 abr 2020]. Disponível em: http://sweetgum.nybg.org/science/ih/

\section{Como citar este artigo \\ How to cite this article}

$(\mathrm{ABNT})$

MANÃO, C. YG.; EDON, D.; MEDEIROS, E. v. S. S.; OLIVEIRA, J. W.; PAIVA, V. F.; SYLVESTRE, L.; LOPES, R. C. Atuação em ensino, pesquisa e extensão no Herbário do Instituto de Biologia da Universidade Federal do Rio de Janeiro. Paubrasilia, Porto Seguro, v. 4, e0060, 2021. DOI 10.33447/paubrasilia.2021.e0060

(Vancouver)

Manão CYG, Edon D, Medeiros EvSS, Oliveira JW, Paiva VF, Sylvestre L, Lopes RC. Atuação em ensino, pesquisa e extensão no Herbário do Instituto de Biologia da Universidade Federal do Rio de Janeiro. Paubrasilia 2021;4:e0060. doi:10.33447/ paubrasilia.2021.e0060 